\title{
Strömgren photometry of the open clusters NGC 6134 and NGC 3680^
}

\author{
H. Bruntt ${ }^{1}$, S. Frandsen ${ }^{1}$, H. Kjeldsen ${ }^{1,2}$, and M.I. Andersen ${ }^{3}$ \\ 1 Institute for Physics and Astronomy, Aarhus University, DK-8000 Aarhus C, Denmark \\ e-mail: bruntt@ifa.au.dk, srf@ifa.au.dk, hans@ifa.au.dk \\ 2 Theoretical Astrophysics Center, Danmarks Grundforskningsfond, Aarhus University, DK-8000 Aarhus C, Denmark \\ 3 Nordic Optical Telescope Apartado 474, Santa Cruz de La Palma, E-38700, Spain \\ e-mail: andersen@not.iac.es
}

Received May 17; accepted June 9, 1999

\begin{abstract}
We present Strömgren photometry of the two southern open clusters NGC 6134 and NGC 3680. The field of NGC 6134 was observed with different shifts of the telescope and rotations of the CCD camera in order to detect and remove any large scale flat field errors using the technique described by Andersen et al. (1995). By using calibrations of the Strömgren indices we find the metallicity and interstellar reddening of the clusters: For NGC 6134 the results are $[\mathrm{Fe} / \mathrm{H}]=0.28 \pm 0.02$ and $E(b-y)=0.263 \pm 0.004$ and for NGC 3680 we find $[\mathrm{Fe} / \mathrm{H}]=0.09 \pm 0.02$ and $E(b-y)=0.048 \pm 0.011$. When comparing the observed colour-magnitude diagrams theoretical isochrones we find that models which include overshooting in the convective cores agree with the extended hook after the turnoff. From the isochrone fit we find ages of $0.69 \pm 0.10$ Gyr and $1.45 \pm 0.15$ Gyr for NGC 6134 and NGC 3680 respectively. Strömgren photometry of six known $\delta$ Scuti stars in NGC 6134 is presented.
\end{abstract}

Key words: techniques: photometric - open clusters and associations: individual: NGC 6134, NGC $3680-\delta$ Sct

\section{Introduction}

Stellar clusters present a unique playground for tests of stellar evolution. Nearby clusters like the Hyades, Pleiades, and Praesepe are especially important due to the possibility to make detailed observations of individual stars, but also open clusters like NGC 3680 (Nordström et al. 1997), IC 4651 (Meibom 1999), and NGC 6134

\footnotetext{
Send offprint requests to: $\mathrm{H}$. Bruntt

* Based on observations made with the Danish 1.54-m telescope at ESO, La Silla, Chile.
}

(Frandsen et al. 1996) have received a lot of attention. In order to do a complete test of stellar evolution providing tight constraints on stellar models, it is necessary to expand the techniques beyond the normal isochrone fitting of models to observed colour-magnitude diagrams. One possibility of expansion is the study of variable stars, in particular $\delta$ Scuti stars, that are members of the clusters.

Among the more distant clusters $(d \geq 1 \mathrm{kpc})$ NGC 6134 still holds the record in terms of the number of $\delta$ Scuti stars, although a fair number has been detected in other clusters (Frandsen et al. 1998; Viskum et al. 1998; Choi et al. 1998).

The southern open cluster NGC 6134 has been observed several times over the last decade. The early study by Kjeldsen \& Frandsen (1989) showed that this cluster was particularly interesting as the location of the turnoff region of the cluster was inside the instability strip. Hence it was expected that several $\delta$ Scuti stars could be found in the turnoff region. Kjeldsen \& Frandsen (1989) found three $\delta$ Scuti stars. The multi site observations of Frandsen et al. (1996) led to the detection of several pulsation frequencies in six $\delta$ Scuti stars and the detection of a variable blue straggler.

To be able to exploit the information contained in the observed pulsation frequencies of the $\delta$ Scuti stars it is extremely important to know the fundamental parameters of the individual cluster stars, i.e. interstellar reddening, metallicity, distance, and age. In this paper we present new Strömgren photometry that leads to much improved values for these parameters.

\section{Observations}

In May 1996 we used the Danish $1.54 \mathrm{~m}$ telescope at La Silla, ESO, to obtain Strömgren photometry of the two 
Table 1. Number of frames obtained of the open clusters NGC 6134 and NGC 3680 in each of the Strömgren filters. The two $\beta$ filters are labeled $\mathrm{W}$ (wide) and $\mathrm{N}$ (narrow)

\begin{tabular}{crrrrrrc}
\hline Cluster & $u$ & $v$ & $b$ & $y$ & $\beta_{\mathrm{W}}$ & $\beta_{\mathrm{N}}$ & Total \\
\hline NGC 6134 & 1 & 19 & 18 & 24 & 10 & 14 & 86 \\
NGC 3680 & 0 & 11 & 17 & 9 & 7 & 8 & 52 \\
\hline
\end{tabular}

open clusters NGC 6134 and NGC 3680 over a period of five nights. We also made observations of 19 bright Strömgren standard stars from the catalogue by Olsen et al. (1994) in order to transform the instrumental magnitudes to standard Strömgren indices.

The combination of the focal reducer instrument Danish Faint Object Spectrograph and Camera (DFOSC) and a Loral/Lesser CCD (W11-4) permits a large area of the clusters to be observed in one frame, i.e. 13.6 by 13.6 arcmin (2048 by 2048 pixels). Due to a failed UV-flooding, the CCD was insensitive in the $u$ band, making the Strömgren photometry incomplete. A large decrease in sensitivity towards the edges was also seen in the remaining filters.

The observations of NGC 6134 were made with several different shifts of the telescope (a few arc minutes) and rotations of the $\mathrm{CCD}$ camera $\left( \pm 90^{\circ}\right)$. This was done in order to detect any dependency of the derived magnitudes on the position of the CCD due to large scale flat field errors. As noted by Andersen et al. (1995) focal reducer instruments, like the DFOSC mounted on the Danish $1.54 \mathrm{~m}$ telescope, may suffer from scattered light problems due to insufficient baffling and/or internal reflection in the optics of the instrument. These effects will result in erroneous flat fields. In Table 1 we note the number of frames obtained in each of the Strömgren filters.

For the observations of the Strömgren standard stars we defocused the telescope in order to get a higher duty cycle. The degree of defocusing was different for each target, i.e. depending on the brightness of each star ( $V$ in the range 9.7 to 7.2 ). We also read out only a small 750 by 400 window on the CCD for these observations.

\section{Basic reductions}

The linearity of the Loral/Lesser CCD was tested by taking dome flat fields with an increasing number of openings of the shutter, i.e. thereby increasing the exposure time but eliminating the finite shutter opening time. Unfortunately, the quartz lamp that was used as a light source was variable at a $5 \%$ level, and hence a non-linearity below this level could not be measured. However, according to the telescope manual (Storm et al. 1996) the CCD is linear below $1 \%$ up to the 16 bit data saturation level of the CCD, which is then our assumed limit for this effect.

The raw science frames were bias subtracted and flat fielded. A weak dependency of the bias level on the ambi-

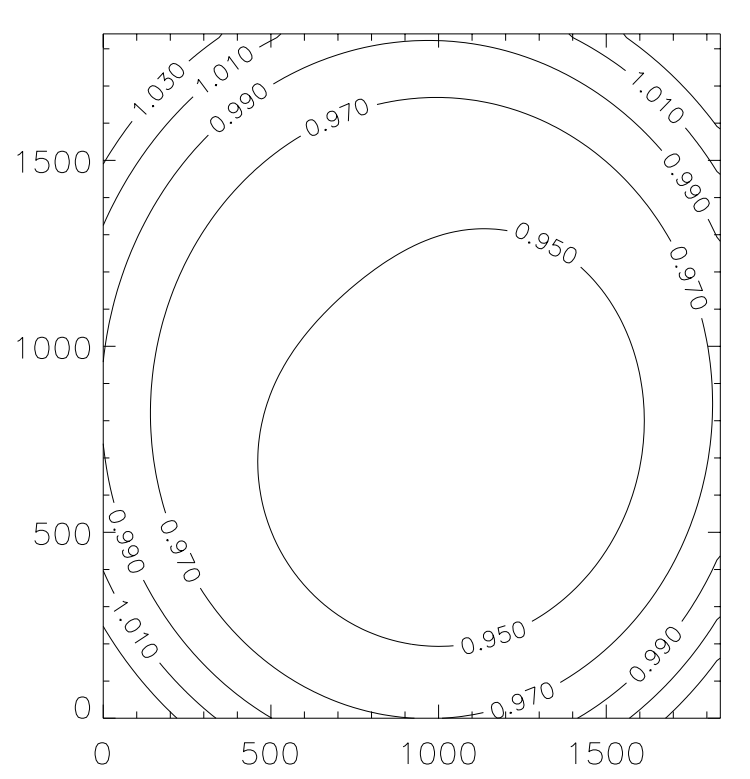

Fig. 1. Contour plot of the flat field correction surface that has been applied to the flat field for the $y$ filter. The abscissa and ordinate show the position (in pixels) on the CCD

ent temperature was determined from several bias frames (0.9 ADU/K). We found that our sky flat fields were not as reliable as the dome flats (different gradients across the field were often seen in two consecutive frames). Hence we decided to use dome flats for the flat fielding procedure. A master flat field for each filter was constructed using the median of several dome flats. The significance of our choice of flat fields is diminished by the flat field correction that is applied later.

After bias subtraction and flat field division the frames of the open clusters were reduced by using the MOMF software package by Kjeldsen \& Frandsen (1992). The MOMF programmes use a combination of PSF- and aperture photometry.

\section{Flat field correction}

The division of a CCD frame by a flat field does not necessarily provide a correct calibration of the CCD sensitivity variation. Errors up to $10 \%$ are seen on some telescopes depending on the baffling and internal reflections in the optics. Andersen et al. (1995) have developed an algorithm, where a correction is derived from a set of shifted and rotated images. It only works for fields with many stars (distant clusters). Using multiple shifted and rotated CCD frames by itself provides some averaging of the flat field errors, but an additional improvement is gained by the technique we have applied. For a more thorough description of the flat field correction the reader is referred to Bruntt (1999). 


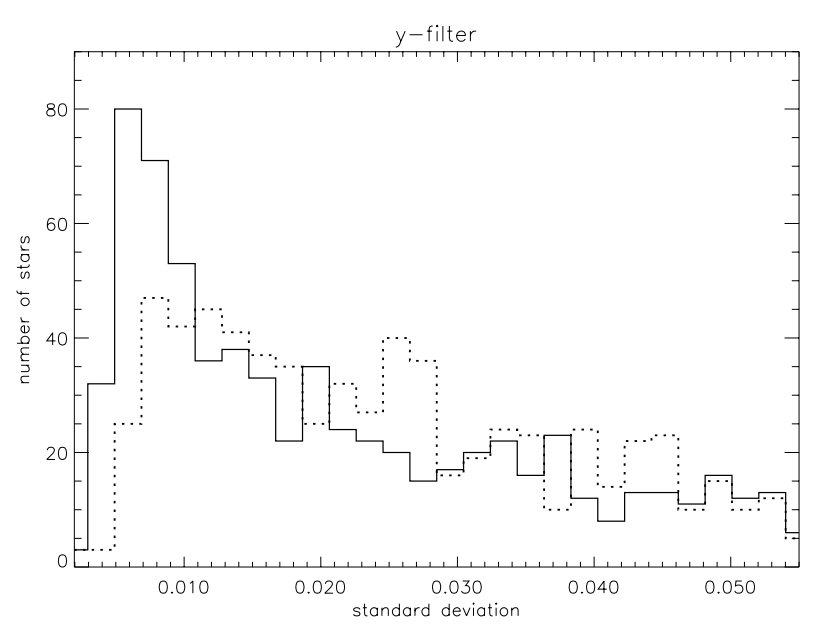

Fig. 2. Histograms of the standard deviation of stars before (dashed) and after (solid) the flat field correction in the $y$ filter calculated from (up to) 22 frames

In Fig. 1 we show a contour plot of the correction surface in the $y$ filter. Note that an error of $\simeq 8 \%$ is found from the centre of the CCD to the extreme edges. In the other Strömgren filters the errors are of the same order of magnitude. This clearly shows that serious problems may be present if one observes a star cluster with only one telescope position.

In Fig. 2 the standard deviation of the brightest stars in the cluster NGC 6134 is seen. The standard deviation is calculated from the magnitudes determined from the (up to) $22 y$ frames.

\section{Standard Strömgren indices}

The flat field correction surfaces in the Strömgren $v b y-\beta$ filters found by using the observations of NGC 6134 have been applied to the observations of both clusters and the Strömgren standard stars.

The atmospheric extinction was determined by using observations of two bright standard stars which were observed as part of a programme to detect oscillations in two bright field stars (see Viskum et al. 1998). These stars were observed at the same time as the open clusters with the Danish $50 \mathrm{~cm}$ SAT telescope at La Silla. From these data we found the extinction coefficients of the standard Strömgren magnitudes, which were applied to the observations of the open clusters.

After the extinction correction we found the following transformation to the standard indices using the Strömgren standard stars:

$$
\begin{aligned}
b-y & =+0.5026+1.0444(b-y)_{I} \\
V & =-1.9027+1.0067 y_{I} \\
m_{1} & =-1.2662+1.0118\left(m_{1}\right)_{I}-0.1291(b-y) \\
\beta & =+0.7277+1.4348 \beta_{I}
\end{aligned}
$$

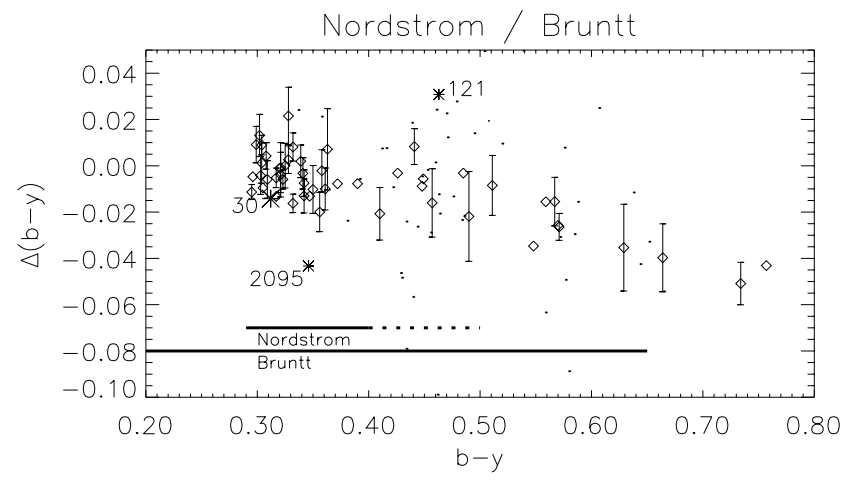

Fig. 3. Comparison of the $b-y$ indices found by Nordström et al. (1997) and the present work. Stars with low standard deviation are plotted with diamonds while other stars are plotted with dots. The double stars " 30 " and " 121 " are marked with stars. The two datasets disagree appreciably for star "2095". The lines labeled "Nordström" and "Bruntt" indicate the range in $b-y$ of the stars used for calibration by Nordström et al. (1997) and in this paper

where $I$ denotes the instrumental index.

We have then compared the derived indices with previous Strömgren photometry of the open cluster NGC 3680. Nissen (1988) has published Strömgren indices from photoelectric measurements of the brightest stars in the field of NGC 3680, i.e. bright field stars and stars at the turnoff region in the cluster. More extended CCD photometric studies of this cluster have been done by Twarog et al. (1989) (uvby) and Nordström et al. (1997) $(b-y)$. Their calibration both rely on the early work by Nissen (1988).

We find small offsets in the $b-y$ and $\beta$ index and in $V$ when comparing with Nissen (1988). The photometry of Nissen is of high quality and is well-calibrated as several $(\sim 30)$ standard stars were observed each night (compared to just five standard stars in our case). Hence we have corrected for the small offset errors, i.e. 0.01 and 0.02 mag in $b-y$ and $\beta$ respectively calculated from 13 and 4 stars in common with Nissen (1988).

In Fig. 3 we show the difference in the $b-y$ index found by Nordström et al. (1997) and the present work. As can be seen there is a significant dependency on colour. Also indicated in Fig. 3 is the range in $b-y$ of the stars used for the standard transformation. Nordström et al. (1997) only have two stars in the dashed range in Fig. 3. As noted by Nordström et al. (1997) their calibration rely on the turnoff stars observed by Nissen (1988), i.e. with only a narrow range in $b-y$. Hence we consider our calibration of the fainter stars in the cluster as being more reliable as our standard stars cover a much more extended range in $b-y$.

The photometric precision of the Strömgren indices are estimated using the standard deviation of the derived instrumental magnitudes. The result for the turnoff (hence brightest) stars in each cluster is shown in Table 2. 
Table 2. Photometric precision of the Strömgren indices in each cluster for stars around the turnoff (TO) and for the F type stars in NGC 6134

\begin{tabular}{lcccc}
\hline Stars & $V$ & $b-y$ & $m_{1}$ & $\beta$ \\
\hline NGC 6134, TO & 0.004 & 0.007 & 0.009 & 0.010 \\
NGC 6134, F & 0.010 & 0.020 & 0.020 & 0.020 \\
NGC 3680, TO & 0.004 & 0.007 & 0.008 & 0.016 \\
\hline
\end{tabular}

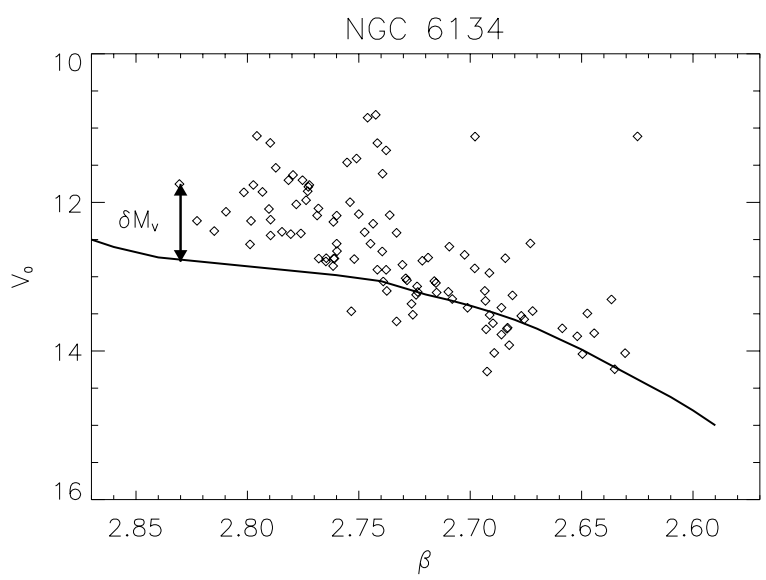

Fig. 4. Conversion of $\delta M_{V}$ to the $c_{0}$ index for stars in NGC 6134. The solid lines are the standard relations found in Crawford (1975a, 1979)

These are A type stars for the younger cluster NGC 6134, while the turnoff stars in NGC 3680 are F type stars. Also quoted is the precision of the F type stars in NGC 6134. The $\mathrm{F}$ type stars are particularly interesting, as their $m_{1}$ index correlates with metallicity. The results found in Table 2 are comparable to what has been achieved earlier with photoelectric equipment, e.g. Nissen (1988).

We have also estimated the standard errors of the Strömgren indices by comparing our results to Nissen (1988) after the removal of offset errors and ignoring $3 \sigma$ outliers. The standard errors (sd. of the mean) for the four indices are 0.0018 (22 stars), 0.0022 (23 stars), 0.0032 (23 stars), 0.0032 (4 stars) in $V, b-y, m_{1}$, and $\beta$ respectively.

\section{The Balmer discontinuity index}

As the CCD was insensitive to light in the $u$ filter we could not obtain the Balmer discontinuity index, $c_{0}=$ $(u-v)-(v-b)$. The calibrations of the interstellar reddening include small terms involving the Balmer discontinuity $c_{0}$ index (Crawford 1975a, 1979), hence it is essential to get a somewhat reliable measure of this index. For NGC 3680 the $c_{0}$ index was obtained for a few stars in the turnoff region by Nissen (1988), and we have used his values.

The $c_{0}$ index measures to what extent a star has evolved above the ZAMS. We can measure the extent of evolution through $\delta M_{V}$ by fitting a ZAMS to the lower end
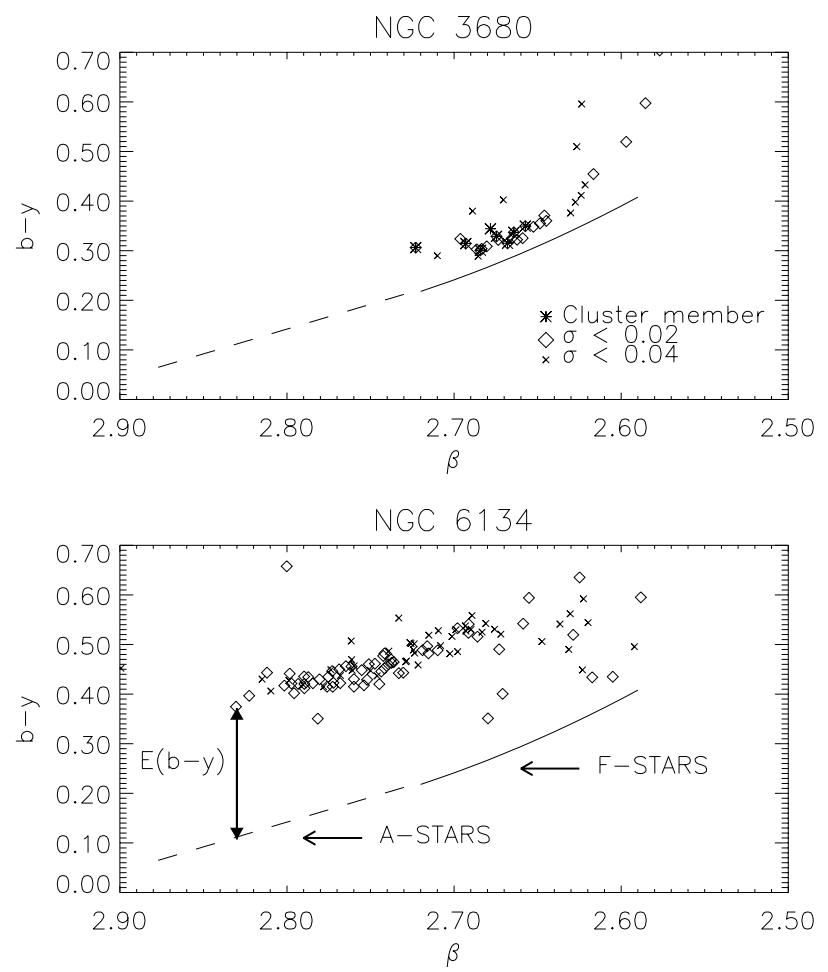

Fig. 5. The relation of $b-y$ and $\beta$ for stars with well-determined indices in NGC 6134 and NGC 3680

of the observed colour-magnitude diagram. For the stars in NGC 6134 we calculated approximate $c_{0}$ indices indirectly by using the calibrations of the $f$ factor by Nissen (1988) (F stars):

$\delta c_{0}=\delta M_{V} / f=\delta M_{V} /(9.0+50(2.72-\beta))$

and Crawford (1979) (A stars):

$\delta c_{0}=\delta M_{V} / f=\delta M_{V} / 9.0$,

where $\delta c_{0}$ is defined as $\delta c_{0}=c_{0}($ ZAMS $)-c_{0}$.

In Fig. 4 we show how $\delta M_{V}$ is converted to the $c_{0}$ index by using the $f$ factor. Note that this was only done for stars with $E(b-y)$ within $3 \sigma$ of the mean value, i.e. stars that are probable cluster members.

\section{Interstellar reddening}

In Fig. 5 we show the $\beta$ index as a function of the $b-y$ index for the observed stars in NGC 6134 and NGC 3680. As can be seen the open cluster NGC 6134 suffers from significant interstellar reddening.

To determine the interstellar reddening we have used the calibrations of Crawford (1975a) (A stars), (1979) (F stars) with a small correction which was noted by Nissen (1988). Histograms of the interstellar reddening for the stars in two open clusters are shown in Fig. 6.

The mean value of each cluster after removing $3 \sigma$ outliers are: NGC 6134: $E(b-y)=0.263 \pm 0.003$ and NGC 3680: $E(b-y)=0.046 \pm 0.011$, cf. Table 3 . 

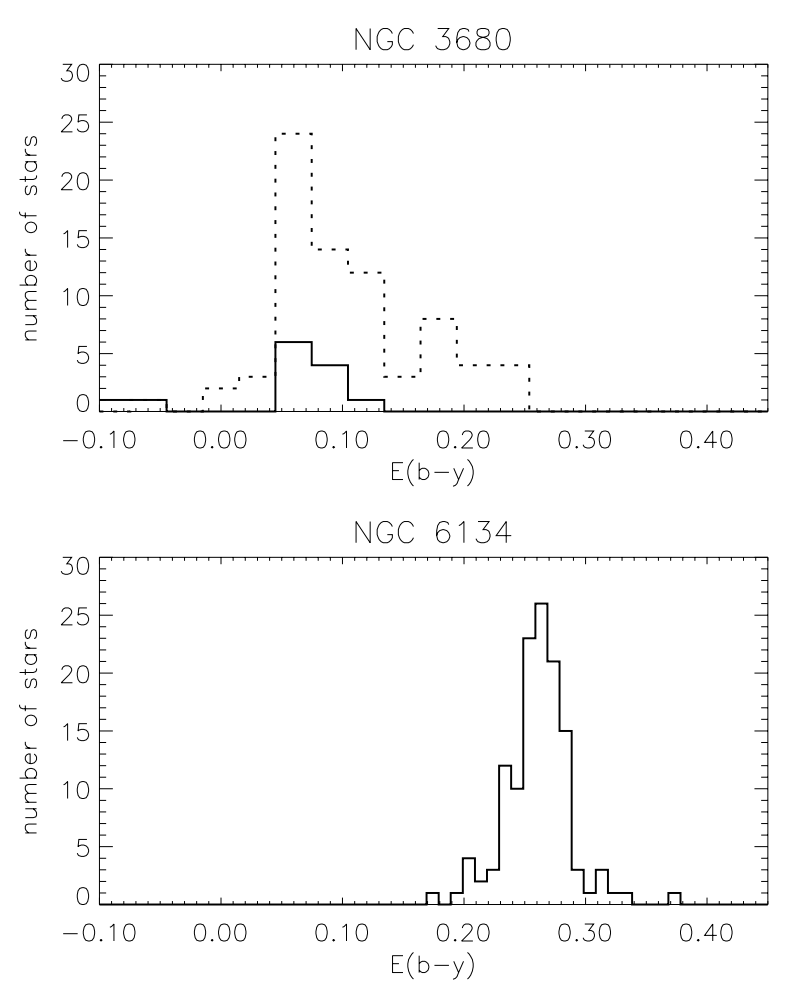

Fig. 6. Histograms of the interstellar reddening of stars in NGC 6134 and NGC 3680. In the top plot the dashed histogram is for all stars, while the solid curve is for the stars that are confirmed cluster members

These results agree reasonably well with previous results obtained for both clusters: For NGC 3680 Nissen (1988) found $E(b-y)=0.034 \pm 0.005$ when using the cluster membership assignment by Nordström et al. (1997). Kozhurina-Platais et al. (1997) found a somewhat higher value $E(b-y)=0.053$ from Johnson $B V$ photometry, when using only stars that were probable cluster members according to proper motion measurements. They made a fit of overshoot isochrones to the observed CMD with $E(b-y)$ and distance modulus as free parameters. Kjeldsen et al. (1991) found $E(b-y)=0.32 \pm 0.02$ for NGC 6134 from Johnson $U B V$ photometry. From revised DDO photometry of giant stars Twarog et al. (1997) estimate that $E(b-y)=0.27$, which is in better agreement with our results. As discussed by Twarog et al. (1997 the reason for the somewhat higher reddening found by Kjeldsen et al. (1991) may be due to the fact that they assumed solar metallicity.

\section{Metallicity}

The reddening corrected $m_{0}$ index is correlated with metallicity for $\mathrm{F}$ type stars. In Fig. 7 we show the relation between the $\beta$ and $m_{0}$ index for stars in each of the observed clusters. Also plotted is the Hyades relation taken from Crawford (1975a, 1979). As may be inferred,
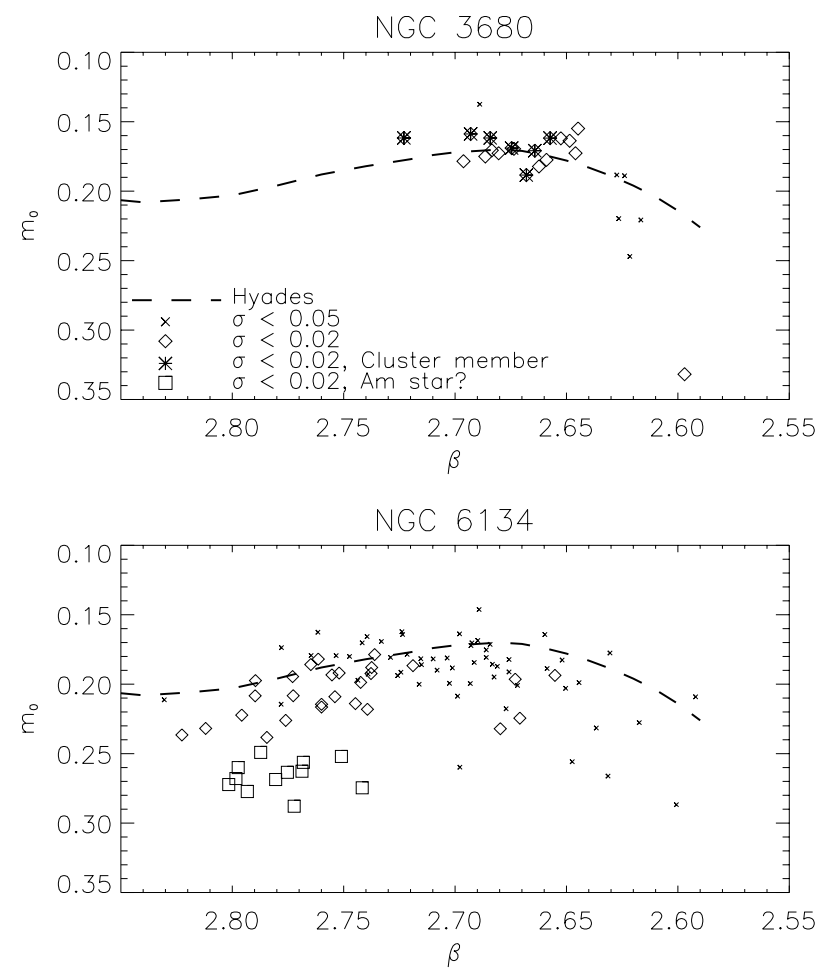

Fig. 7. The relation between $m_{0}$ and $\beta$ for stars in NGC 3680 (top) and NGC 6134 (bottom). Also plotted is the standard Hyades relation taken from Crawford (1975a, 1979). The symbols are explained in the top plot

the metallicity of NGC 3680 is somewhat lower than the Hyades cluster, while the metallicity of NGC 6134 is somewhat higher. Note that $[\mathrm{Fe} / \mathrm{H}]_{\text {Hyades }} \simeq 0.12$ according to recent observations by Cayrel (1985).

In the field of NGC 6134 a few of the F type stars (i.e. $\beta<2.72$ ) have high $m_{0}$ indices, which indicate that they are not members of the cluster. On the other hand it can also be seen that quite a few of the A stars in NGC 6134 also have extremely high $m_{0}$ indices, which not necessarily exclude them from being cluster members, but may indicate that they are Am type stars. Note that they are all found on the upper part of the cluster main sequence. We have used the calibration of $[\mathrm{Fe} / \mathrm{H}]$ for $\mathrm{Am}$ stars by Smalley et al. (1993) on the possible Am stars (i.e. $\delta m_{0}<-0.05$ and $\left.\beta>2.72\right)$ in the cluster. We find a mean metallicity of $[\mathrm{Fe} / \mathrm{H}]=0.83 \pm 0.04$ (standard deviation of mean) from 12 possible Am stars.

A calibration of the $m_{0}$ index with metallicity has been obtained by Nissen (1981). In Fig. 8 we have plotted the histogram of stars in NGC 6134 which are probable cluster members, i.e. stars with interstellar reddening within $3 \sigma$ of the mean value. For NGC 3680 we have plotted the result for 12 stars which are cluster members according to the spectroscopy by Nordström et al. (1997). The mean value of each cluster is found by removing $3 \sigma$ outliers. 

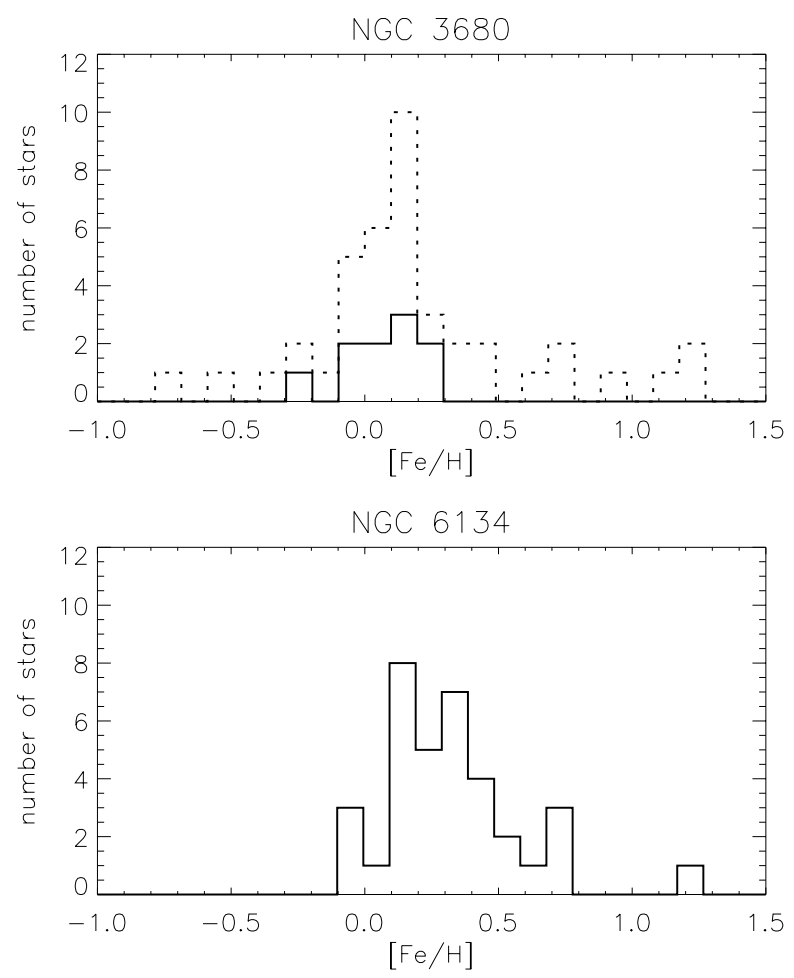

Fig. 8. Histograms of the metallicity for stars in the field of NGC 6134 and NGC 3680. In the top plot the dashed line includes all stars, while the solid line is for the confirmed cluster members

Our results are: NGC 6134: $[\mathrm{Fe} / \mathrm{H}]=0.28 \pm 0.02$ and NGC 3680: $[\mathrm{Fe} / \mathrm{H}]=0.09 \pm 0.02$, cf. Table 3 .

The uncertainty of the metallicity may be as large as $0.1-0.2$ dex for NGC 6134, as only few Strömgren standard stars with high $b-y$ were included in the calibration. This is a problem as the F stars in NGC 6134 are highly reddened. The result for NGC 6134 agrees with what is given in the catalogue by Lyngå $(1987)[\mathrm{Fe} / \mathrm{H}]=0.25$. Twarog et al. (1997) have found $[\mathrm{Fe} / \mathrm{H}]=0.18 \pm 0.09$ from revised DDO photometry. On the other hand Claria et al. (1992) found $[\mathrm{Fe} / \mathrm{H}]=-0.05 \pm 0.12$ from photometry of red giant stars in the cluster. They used three different photometric methods, $U B V$, DDO and Washington which yielded $[\mathrm{Fe} / \mathrm{H}]=0.08,-0.16$, and -0.11 respectively.

Several estimates of the metallicity of NGC 3680 have been published. Nordström et al. (1997) found $[\mathrm{Fe} / \mathrm{H}]=$ $0.11 \pm 0.05$ when using their cluster membership assignment and the Strömgren photometry of Nissen (1988). Twarog et al. (1997) cite $[\mathrm{Fe} / \mathrm{H}]=-0.12 \pm 0.05$ from DDO photometry of 8 giant stars.

It is apparent from the published results that it is difficult to determine the metallicities within 0.15 dex by means of photometry. A determination of the metallicities by using spectroscopy would certainly be of great value.
Table 3. Fundamental parameters of NGC 6134 and NGC 3680: interstellar reddening, metallicity, age (Gyr), and distance $(\mathrm{kpc})$. The estimated standard errors are given in parenthesis

\begin{tabular}{ccccc}
\hline Cluster & $E(b-y)$ & {$[\mathrm{Fe} / \mathrm{H}]$} & Age & $d$ \\
\hline NGC 6134 & 0.263 & 0.28 & 0.69 & 1.41 \\
& $(0.004)$ & $(0.02)$ & $(0.10)$ & $(0.06)$ \\
NGC 3680 & 0.048 & 0.09 & 1.48 & 1.10 \\
& $(0.011)$ & $(0.02)$ & $(0.15)$ & $(0.05)$ \\
\hline
\end{tabular}

\section{Colour-magnitude diagrams}

The observed colour-magnitudes diagrams are seen in Fig. 9. Note that the $V$ magnitude and the $b-y$ index have been corrected for interstellar extinction $\left(A_{V}=\right.$ $4.3 E(b-y))$. In each diagram 606 (NGC 6134) and 164 (NGC 3680) stars are plotted. As the metallicity of the clusters are comparable it is evident that NGC 6134 is younger than NGC 3680, i.e. no A type stars are seen in the CMD of NGC 3680.

\section{Ages and distances}

We have used theoretical isochrones calculated using the recent programme by Pols et al. (1998). We have fitted isochrones which rely on standard physics and models which also include overshooting. The programme by Pols et al. (1998) provides $V$ and $B-V$ colours which rely on synthetic spectra by Kurucz et al. (1992). The conversion from the observed Strömgren indices to effective temperatures is made by applying the calibration of Alonso et al. (1996). Note that this calibration includes a term proportional to $c_{0}$, hence only stars for which the $c_{0}$ index have been inferred are plotted (as described in Sect. 6).

We calculate a range of isochrones with the metallicity found in Sect. 8. The isochrones are shifted vertically to fit the lower end of the main sequence, and then the age is changed to fit the turnoff stars. The result for both standard models (dashed line) and overshooting models (solid line) is shown in Fig. 10.

It seems that the isochrones from models including overshoot describe the extended hook in NGC 6134 better than the standard model isochrones. No firm conclusion can be given here for NGC 3680 from the limited sample of stars. However, previous studies of this cluster by Nordström et al. (1997) (Strömgren by photometry) and Kozhurina-Platais et al. (1997) (BV photometry) both agree that isochrones that include some degree of overshoot are needed to explain the observed CMD.

The derived fundamental parameters of the open clusters using the Strömgren indices (metallicity and interstellar reddening) and the age and distance found when using isochrones that include overshoot are quoted in Table 3.

Nordström et al. (1997) have performed isochrone fitting using several models, and also found that isochrones 

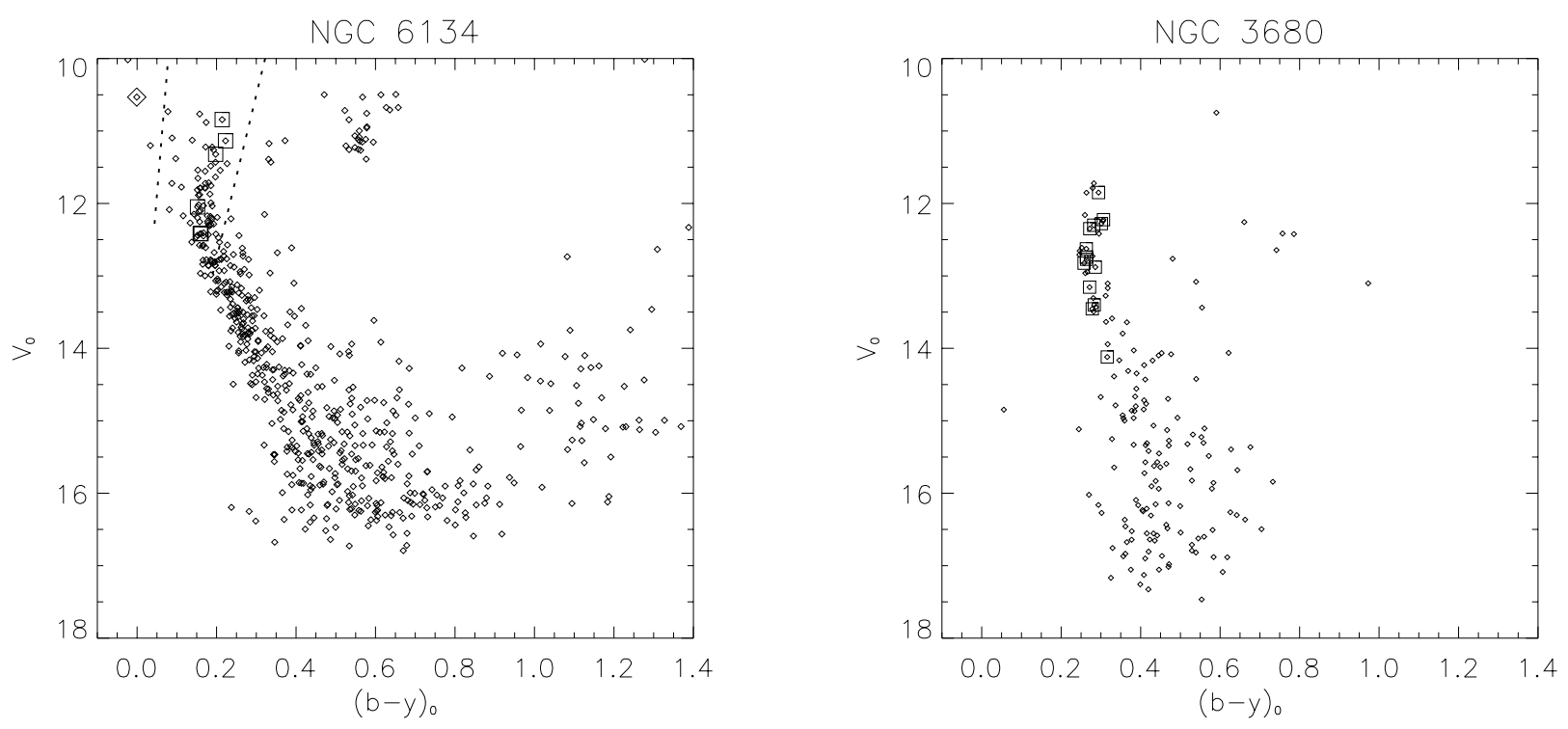

Fig. 9. Observed colour-magnitude diagrams of NGC 6134 and NGC 3680. In the CMD of NGC 6134 the boxes mark the location of the six known $\delta$ Scuti stars and the dashed curves show the location of the instability strip according to observations of field stars by Rodriguez et al. (1996). The diamond marks the blue straggler found by Frandsen et al. (1996). In the CMD of NGC 3680 the boxes mark the stars that are cluster members according to Nordström et al. (1997)
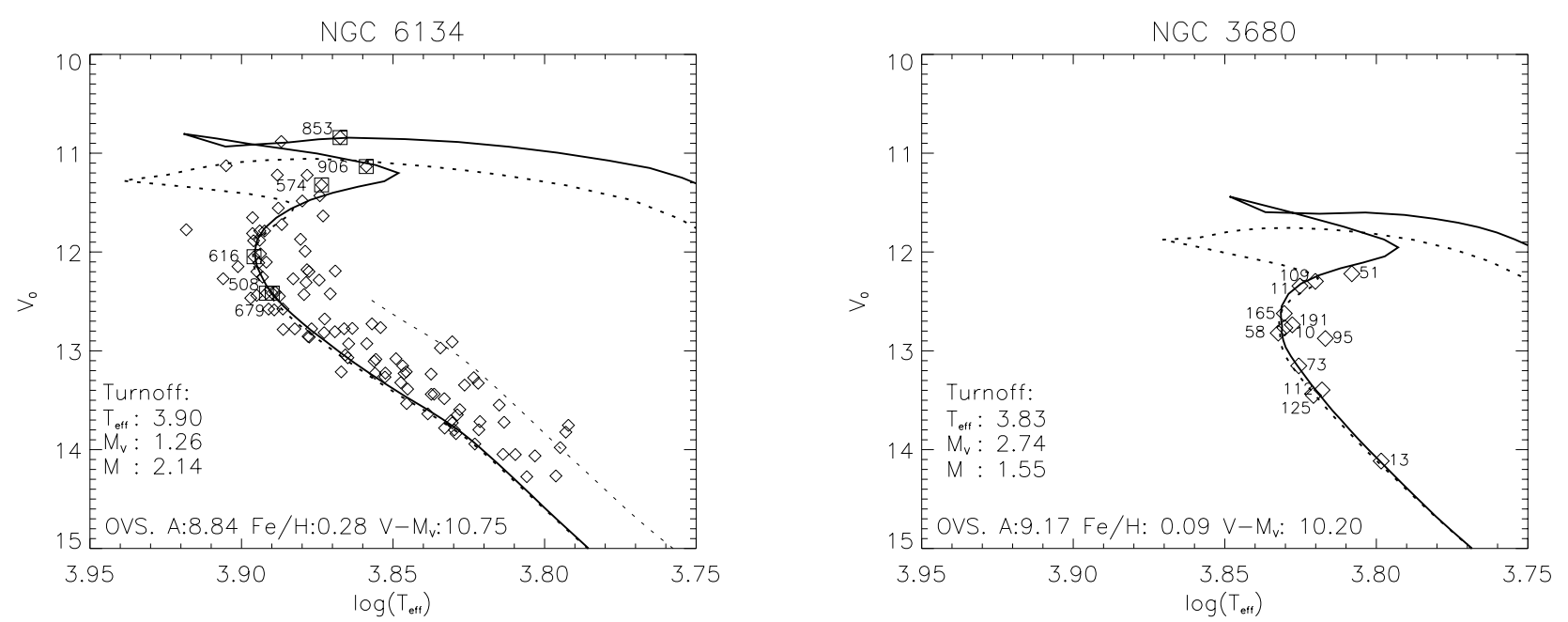

Fig. 10. The figures show Pols theoretical isochrones that best fit NGC 6134 and NGC 3680. Dashed lines are standard models while solid lines are models which include overshooting. In the left figure is also plotted a part of the standard model isochrone when shifted -0.75 in $V$, i.e. the approximate location of the binary star sequence. In the plot to the left the box symbols mark the $\delta$ Scuti stars. In both plots are given the parameters of the model at the turnoff for the overshoot isochrones $\left(T_{\text {eff }}, M_{V}\right.$, and $\left.M / M_{\odot}\right)$ and log age $(A)$, metallicity $([\mathrm{Fe} / \mathrm{H}])$, and distance modulus $\left(V-M_{V}\right)$

Table 4. Astrophysical parameters for the six $\delta$ Scuti stars in NGC 6134

\begin{tabular}{rrcccccccc}
\hline $\mathrm{ID}_{\mathrm{B}}$ & $\mathrm{ID}_{\mathrm{F}}$ & $V$ & $b-y$ & $m_{1}$ & $\beta$ & $E(b-y)$ & $M / M_{\odot}$ & $T_{\text {eff }}$ & $L / L_{\odot}$ \\
\hline 508 & 397 & 13.548 & 0.422 & 0.159 & 2.784 & 0.256 & 1.99 & 7795 & 18.0 \\
574 & 348 & 12.452 & 0.461 & 0.104 & 2.738 & 0.271 & 2.41 & 7476 & 49.4 \\
616 & 159 & 13.179 & 0.415 & 0.136 & 2.778 & 0.252 & 2.13 & 7869 & 25.3 \\
679 & 87 & 13.547 & 0.424 & 0.144 & 2.758 & 0.236 & 1.99 & 7760 & 18.0 \\
853 & 161 & 11.973 & 0.477 & 0.120 & 2.742 & 0.264 & 2.47 & 7370 & 76.8 \\
906 & 9 & 12.266 & 0.486 & 0.181 & 2.698 & 0.227 & 2.46 & 7226 & 58.7 \\
\hline
\end{tabular}


which include some amount of overshooting are needed. They find an age of $1.45 \pm 0.3$ Gyr when fitting an overshoot isochrone to 12 stars around the turnoff, which is in excellent agreement with our result, i.e. age $1.48 \pm 0.3 \mathrm{Gyr}$. Nordström et al. (1997) determine the distance by fitting the Hyades cluster to the lower main sequence of NGC 3680 and find $V-M_{V}=10.5 \pm 0.3$, which agrees with our somewhat smaller value $V-M_{V}=10.2 \pm 0.2$, which was found by shifting the isochrone to fit the lower main sequence. Kozhurina-Platais et al. (1997) found age $1.6 \pm 0.15 \mathrm{Gyr}$ and $V-M_{V}=10.2 \pm 0.3$. Note that they used a somewhat higher value for the interstellar reddening and also used solar metallicity isochrones. It is important to note that the present results and those by Nordström et al. (1997) and Kozhurina-Platais et al. (1997), are obtained by using different isochrone codes. It is reassuring that our results for the age and distance of NGC 3680 agree within the errors.

Kjeldsen \& Frandsen (1991) fitted standard model isochrones to their observed CMD of NGC 6134 and found distance modulus $V-M_{V}=11.25$ and age 0.89 Gyr which we will now compare with our results, i.e. $V-M_{V}=$ $10.75 \pm 0.20$ and age $0.69 \pm 0.10$. Twarog et al. (1997) have determined $V-M_{V}=11.1$ when using the $B V$ photometry by Kjeldsen \& Frandsen (1991) but with different interstellar reddening (Sect. 7) and metallicity (Sect. 8).

The reason for the somewhat higher distance modulus found by Kjeldsen \& Frandsen (1991) is only partly explained by the somewhat lower value of $E(b-y)$ found by us, yielding a difference in $V$ of $\Delta V=4.3 \times 0.059=$ 0.25. Kjeldsen \& Frandsen (1991) used Solar metallicity isochrones, i.e. isochrones with somewhat higher $M_{V}$ and higher $T_{\text {eff }}$ (fixed age). On the other hand this was standard isochrone models, i.e. isochrones with higher $M_{V}$ and lower $T_{\text {eff }}$ (fixed age). Consequently, these differences alone do not explain the large difference in distance modulus or age. We note that Kjeldsen \& Frandsen (1991) only had a very limited sample of $\sim 60$ stars. They also had no individual determination of the interstellar reddening, as we now have, in order to exclude stars that are clearly not members of the cluster. The difference in the quality and quantity of the two data sets explain the differences in the determined age and distance.

\section{The $\delta$ Scuti stars in NGC 6134}

In Table 4 we present the fundamental astrophysical parameters for the six $\delta$ Scuti stars in NGC 6134, which has been derived from the Strömgren photometry. These data will be of great importance when it comes to the detailed modeling of these stars. ID F $_{\mathrm{F}}$ are the star numbers in Frandsen et al. (1996). $M / M_{\odot}$ are the estimated masses found by interpolation to the best fitting isochrone which includes overshoot. $T_{\text {eff }}$ is found by using the calibration by Alonso et al. (1996). $L / L_{\odot}$ is found by using the solar value $M_{\mathrm{bol}}=4.75$, a bolometric correction of $B C=-0.06$ and the cluster parameters (distance and interstellar reddening) from Table 3.

\section{Conclusions and prospects}

The new Strömgren photometry of the two open clusters NGC 6134 and NGC 3680 have provided the following information:

1. Large scale flat field errors were removed by taking shifted and rotated frames of NGC 6134. This improves the individual parameters for bright stars, e.g. the known $\delta$ Scuti stars in the cluster.

2. We have made an improved calibration of the lower end of the main sequence for the well studied cluster NGC 3680.

3. We have obtained reliable estimates of the interstellar reddening of the clusters. For NGC 6134 this has been used to determine which stars are clearly not members of the cluster.

4. The approximate metallicity of the clusters have been found.

5. In NGC 6134 several Am type stars are found.

6. By using theoretical isochrones we show that models including overshooting are needed to fit the extended hook of the observed colour-magnitude diagram of NGC 6134.

Our results for the interstellar reddening, metallicity, age, and distance of each cluster agree reasonably well with recent published results. Still, reliable values for the metallicities of the clusters are missing. Attempts have been made by using DDO photometry of giant stars and Strömgren photometry of F type stars, which yield quite different results. The metallicity must be obtained by using spectroscopy which would also be valuable for determining the global properties of these clusters, i.e. age and distance.

The results presented here are perhaps most interesting for NGC 6134, as six $\delta$ Scuti stars have been found in this cluster by Frandsen et al. (1996). In principle we can extract new information contained in the observed pulsation frequencies and amplitudes by constructing theoretical models of the variable stars. The information needed is:

- Pulsation frequencies and amplitudes (multi site timeseries photometry).

- The fundamental parameters of the cluster (Strömgren photometry and spectroscopy).

- Rotational velocities (spectroscopy).

By now we have two of the three needed pieces in the puzzle! Still, the metallicity is unreliable, and a better determination is needed, e.g. by using spectroscopy. We have been allocated observing time for doing spectroscopy of the brighter stars in the two clusters. These observations 
hopefully provide a complete set of parameters for the $\delta$ Scuti stars in NGC 6134 making this cluster one of the key clusters for tests of stellar evolution.

The photometry is available at the CDS database in Strasbourg and through anonymous ftp at aauobs.ifa.au.dk in the /pub/bruntt directory.

Acknowledgements. This research was supported by the Danish Natural Science Research Council through its Centre for Ground-based Observational Astronomy and by the Danish National Foundation through its establishment of the Theoretical Astrophysics Center. We thank Tim Bedding for reading our manuscript. We are grateful to Erik Heyn Olsen who provided us with an updated list of the observed standard stars.

\section{References}

Alonso A., et al., 1996, A\&A 313, 873

Andersen M.I., Freyhammer L., Storm J., 1995, in Calibrating and Understanding HST and ESO instruments, ESO Conference and Workshop Proceedings, Nos. 53, 87, Bienvenuti P. (ed.)

Bruntt H., 1999, Master Thesis, Institute of Physics and Astronomy, Aarhus University

Cayrel R., et al., 1985, A\&A 146, 249

Choi H.S., Kim S.-L., Kang Y.H., 1998, IBVS 4545

Crawford D.L., 1975, AJ 80, 955 (Calibration of F-G stars)

Crawford D.L., 1979, AJ 84, 1858 (Calibration of A stars)

Frandsen S., Balona L.A., Viskum M., Koen C., Kjeldsen H., 1996, A\&A 308, 132
Frandsen S., Arentoft T., 1998, A\&A 333, 524

Kjeldsen H., Frandsen S., 1989, A\&A 215, 287

Kjeldsen H., Frandsen S., 1991, A\&AS 87, 119

Kjeldsen H., Frandsen S., 1992, PASP 104, 413

Kozhurina-Platais V., Girard T.M., Platais I., van Altena W.F., 1997, AJ 109, 672

Kurucz R.L., 1992, Proc. IAU Symp. 149, 225

Lyngå 1987, Catalogue of Open clusters, Lund Observatory

Meibom S., 1999, Master Thesis, Niels Bohr Institute for Astronomy, Physics, and Geophysics, University of Copenhagen

Claria J.J., Mermilliod J.C., 1992, A\&AS 96, 429

Nissen P.E., 1981, A\&A 97, 145

Nissen P.E., 1988, A\&A 199, 146

Nordström B., Andersen J., Andersen M.I., 1997, A\&A 322, 460

Olsen E. Heyn, Perry C.L., 1994, A\&A 104, 429, and references therein. Updated values of the observed standard stars (private communication)

Pols O., Schröder K.P., Hurley J.R., Tout C.A, Eggleton P.P., 1998, MNRAS 298, 525

Rodriguez E., et al., 1996, A\&A 106, 21

Smalley B., Dworetsky M.M., 1993, ASP Conf. Ser. 44, Dworetsky M.M., Castelli F., Faraggiana R. (eds.)

Storm J., Reipurth B., Augusteijn A., 1996, User's Manual for TCS and DFOSC

Twarog B.J.A., Twarog B.A., Shodhan S., 1989, AJ 98, 1634

Twarog B.A., Ashman K.M., Anthony-Twarog B.J., 1997, AJ 114,2556

Viskum M., Hernández M.M., Belmonte J.A., Frandsen S., 1998, A\&A 328, 158

Viskum M., Kjeldsen H., Bedding T.R., Dall T.H., Baldry I.K., Bruntt H., Frandsen S., 1998, A\&A 335, 549 